\title{
A Three-Dimensional Iterative Scheme for an Electromagnetic Capacitive Applicator
}

\author{
MACIEJ J. SOWIŃSKI AND PETER M. VAN DEN BERG
}

\begin{abstract}
An efficient iterative method for solving quasi-static electromagnetic field problems is presented. A relaxation function is introduced in the quasi-static field equations. Then, the resulting equations can be solved by iteration. The method is similar to the one of solving a Laplace equation by computing the stationary state of a diffusion equation. Next, for a radially layered configuration the numerical results are compared with the results from an existing integral equation method. Subsequently, for a realistic three-dimensional model of a human knee numerical results are arrived at.
\end{abstract}

\section{INTRODUCTION}

A RING capacitor applicator has recently been developed [1] for regional deep heating in human tissue. With this type of applicators it seems possible to create a sufficient amount of electromagnetic heat disposition in deep-seated parts of human body.

For a radially layered configuration a computer model has been presented [2] based on an integral equation method of the relevant quasi-static electromagnetic problem. Numerical results have been presented and agreement with experimental results has been established. However, for an arbitrary three-dimensional configuration this computer model cannot be employed.

In the present paper we discuss a method based on the direct discretization of the governing partial differential equations. In order to handle the large number of unknowns, an iterative method was developed to solve the discretized equations. By introducing a spatially dependent relaxation function and an iteration coordinate the problem may be solved by iteration. The method is similar to the one of solving a Laplace equation from a parabolic (diffusion-type) partial differential equation by using an explicit, stable scheme [3]. When we have reached the stationary state we have solved our quasi-static problem.

If we choose our relaxation function to be constant, we end up with a particular form of the "successive overrelaxation" method [4]. The introduction of a spatially

Manuscript received July 10, 1989; revised November 16, 1989. This work was supported by Enraf-Nonius Company, Delft, The Netherlands.

M. J. Sowiński is with the Laboratory for Electromagnetic Research, Department of Electrical Engineering, Delft University of Technology, Delft, The Netherlands, on leave from the Department of Electromagnetic Wave Theory, Institute of Fundamental Technological Research, Warsaw, Poland.

P. M. van den Berg is with the Laboratory for Electromagnetic Research, Department of Electrical Engineering, Delft University of Technology, Delft, The Netherlands.

IEEE Log Number 9037727. dependent relaxation function in our iterative scheme leads to an iterative method superior to this successive overrelaxation method. For a radially layered configuration, the numerical results are compared with the results of an integral equation method [2]. Good agreement has been achieved. We finally present the numerical results for a realistic, three-dimensional model of the human knee. In a number of cross sections we present the electromagnetic (EM) power distribution.

\section{Formulation}

The configuration to be investigated is shown in Fig. 1. A pair of ring-shaped, perfectly conducting electrodes is located around a tissue configuration and fed by a sinusoidally in-time varying voltage source. The complex time representation of field quantities is used with time factor $\exp (j \omega t)$ where $\omega=2 \pi f(f=$ frequency of operation $)$.

The electromagnetic properties of the media are characterized by its permittivity $\epsilon$ and electrical conductivity $\sigma$. In the range of frequencies ( $f \leq 30 \mathrm{MHz}$ ) the quasistatic approximation, $\nabla \times E=0$, is valid and hence the electric field $\boldsymbol{E}$ can be written as

$$
\boldsymbol{E}=-\nabla V=-\left(i_{x} \partial_{x}+i_{y} \partial_{y}+i_{z} \partial_{z}\right) V(x, y, z),
$$

where $V$ is the potential, while $i_{x}, i_{y}, i_{z}$ are unit vectors in Cartesian coordinate system. From the generalized electric current density $\boldsymbol{J}$ and the continuity equation

$$
\begin{aligned}
\nabla \cdot \boldsymbol{J} & =\partial_{x} J_{x}+\partial_{y} J_{y}+\partial_{z} J_{z}, \\
\boldsymbol{J} & =(\sigma+j \omega \epsilon) \boldsymbol{E}
\end{aligned}
$$

together with (1), we obtain a system of two partial differential equations to be satisfied by the electric potential and generalized current

$$
\begin{aligned}
\nabla \cdot \boldsymbol{J} & =0, \\
\boldsymbol{J} & =-\kappa(x, y, z) \nabla V, \\
\kappa(x, y, z) & =\sigma(x, y, z)+j \omega \epsilon(x, y, z) .
\end{aligned}
$$

The volume density of dissipated electromagnetic (EM) power can be obtained from the relation

$$
\begin{aligned}
\dot{w}(x, y, z) & =\frac{1}{2} \sigma(x, y, z)\left[|\boldsymbol{E}(x, y, z)|^{2}\right] \\
& =\frac{1}{2} \frac{\sigma(x, y, z)}{|\kappa(x, y, z)|^{2}}\left[|J(x, y, z)|^{2}\right]
\end{aligned}
$$

0018-9294/90/1000-0975\$01.00 ㄷ 1990 IEEE 


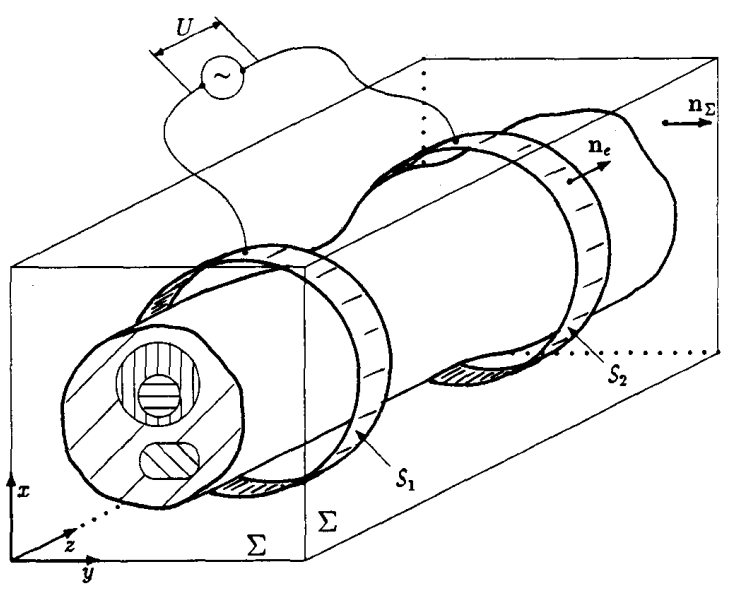

Fig. 1. The applicator configuration.

where in the second expression of (4) we have used (2b). To determine the field in the whole domain shown in Fig. 1 , we must further specify the boundary conditions at the interfaces. At the electrode surfaces $S_{1}$ and $S_{2}$ the electric potential is constant, viz.,

$$
V(x, y, z)= \begin{cases}V_{1}, & (x, y, z) \in \mathrm{S}_{1}, \\ V_{2}, & (x, y, z) \in \mathrm{S}_{2},\end{cases}
$$

while at all other interfaces, the potential $V$ and the component of the current $\boldsymbol{J}$ normal to the interface are continuous. The value of the electric current fed into one of the electrodes by an external source follows from the surface integral of the normal component of the current density evaluated over whole electrode surface,

$$
I_{q}=\iint_{\delta_{q}} \boldsymbol{J} \cdot \boldsymbol{n}_{e} d s
$$

where $\boldsymbol{n}_{e}$ is an outward normal vector to the electrode surface $S_{q}$. The electrodes are fed in such a way that the difference between the potentials $V_{1}$ and $V_{2}$ is equal to the output voltage of the power feeding generator,

$$
U=V_{1}-V_{2} \text {, }
$$

and the net current fed through both electrodes vanishes,

$$
I_{1}+I_{2}=0 \text {. }
$$

To solve our problem, we have to truncate our domain of investigation. We assume some external boundary surface $\Sigma$ where we prescribe an impedance boundary condition of the type,

$$
V=\beta(x, y, z) J \cdot n_{\Sigma}, \quad(x, y, z) \in \Sigma
$$

where $\boldsymbol{n}_{\Sigma}$ is an outward normal vector to $\Sigma$ and $\beta$ is a spatially dependent specific impedance at the point $(x, y$, $z$ ) of the surface $\Sigma$. This boundary condition is the quasistatic version of the radiation boundary condition. In our case, it allows us to control the outflow of the generalized electric current $\boldsymbol{J} \cdot \boldsymbol{n}_{\Sigma}$ through the surface $\Sigma$. In particular, for $\beta=0$ we get from (9) that $V=0$ at the surface $\Sigma$ and this means that $\Sigma$ comes to play the role of the third electrode with potential $V_{\mid \Sigma}=0$. For $\beta=\infty$ the current $J$. $\boldsymbol{n}_{\Sigma}$ should be equal to zero what means that there is no outflow of energy through the surface $\Sigma$.

\section{General Concept of the Iterative Method}

To work with strongly inhomogeneous media and to handle the large number of unknowns in a three-dimensional problem we design a suitable iterative method for the problem formulated above. To obtain this iterative process we extend our three-dimensional problem to a four-dimensional one. The new fourth coordinate is the iteration coordinate $\tau$. The general definition of the convergence of such a process has the form

$$
\begin{aligned}
\lim _{\tau \rightarrow \infty} V(x, y, z ; \tau) & =V_{\infty}(x, y, z), \\
\lim _{\tau \rightarrow \infty} J(x, y, z ; \tau) & =J_{\infty}(x, y, z), \\
\lim _{\tau \rightarrow \infty}\left[V_{1}(\tau)-V_{2}(\tau)\right] & =U,
\end{aligned}
$$

where the pair $V_{\infty}, J_{\infty}$ together with potential difference $U$ between the electrodes is the desired solution to our three-dimensional problem.

Let us define our present problem of (3), (5), (7), (8), and (9) as the initial boundary value problem in a fourdimensional domain with an iteration coordinate $\tau$, as the fourth coordinate, viz.,

$$
\begin{aligned}
\frac{\partial V}{\partial \tau} & =-F(x, y, z) \nabla \cdot \boldsymbol{J}(x, y, z ; \tau), \\
\boldsymbol{J} & =-\kappa(x, y, z) \nabla V(x, y, z ; \tau),
\end{aligned}
$$

with impedance boundary conditions (9), with boundary conditions at electrode surfaces $S_{1}$ and $S_{2}$

$$
V(x, y, z ; \tau)= \begin{cases}V_{1}(\tau), & (x, y, z) \in S_{1}, \\ V_{2}(\tau), & (x, y, z) \in S_{2} .\end{cases}
$$

$$
U=V_{1}(\tau)-V_{2}(\tau),
$$

$$
\lim _{\tau \rightarrow \infty}\left(I_{1}(\tau)+I_{2}(\tau)\right)=0,
$$

and with initial value condition

$$
\begin{aligned}
V(x, y, z ; 0) & =V_{0}(x, y, z), \\
J(x, y, z ; 0) & =J_{0}(x, y, z), \\
V_{q}(0) & =(-1)^{q} \frac{U}{2}, \quad q=1,2 .
\end{aligned}
$$

Furthermore, the relaxation function $F$ is a suitably chosen function such that

$$
F(x, y, z) \neq 0 \quad \text { for each }(x, y, z) \text {. }
$$


The four-dimensional initial boundary value problem formulated above usually has a solution which possesses the property of reaching some equilibrium solution [cf. (10)], when $\tau \rightarrow \infty$. Note, that for real functions $F$ and $\kappa$, and for fixed and real $V_{1}$ and $V_{2},(11 \mathrm{a})$ and (11b) describe a diffusion problem with boundary conditions (5) and (9), and initial conditions (13a) and (13b).

One of the possible solutions to our four-dimensional problem is the "marching-on" process in $\tau$ coordinate. In this general form of an iterative method we assume that we have obtained a field solution for $\tau=\tau_{n}$, and then, using the information contained in this solution, we want to obtain the field values for $\tau=\tau_{n+1}$. In Section $\mathrm{V}$, we discuss this iterative procedure in more detail. If the equilibrium $(\partial V / \partial \tau=0, \tau \rightarrow \infty)$ is achieved, the iterative process described in this way has been converged to the desired solution of the original three-dimensional problem defined by (3), (5), (7), (8), and (9).

As the next step we discuss a proper numerical discretization of our problem described by (11)-(14).

\section{Finite Difference Approximation in Space}

Let us introduce in the spatial domain a rectangular grid with mesh sizes $h_{x}, h_{y}, h_{z}$. The discretized space contains the body shown in Fig. 1, including the electrodes and some free space outside it. The nodal points are located in the baricenters of each elementary block as shown in Fig. 2.

Integrating (11a) over the volume $\nabla_{k, l, m}=h_{x} h_{y} h_{z}$ of an elementary block and assuming that $V$ is constant inside the block and equal to $V_{k, l, m}$ we arrive at

$$
\left(\frac{\partial V}{\partial \tau}\right)_{k, l, m}=\frac{-1}{h_{x} h_{y} h_{z}} \iint_{v_{k, l, m}} \int_{j} F \cdot J d v .
$$

Assuming that the relaxation function $F$ is constant in each block we can apply Gauss' theorem to (15) and arrive at

$$
\left(\frac{\partial V}{\partial \tau}\right)_{k, l, m}=\frac{-F_{k, l, m}}{h_{x} h_{y} h_{z}} \iint_{s_{k, l, \mathrm{~m}}} \boldsymbol{J} \cdot \boldsymbol{n} d s,
$$

where $S_{k, l, m}$ is the surface enclosing the elementary block with nodal point $(k, l, m) . F_{k, l, m}$ is the value of the relaxation function $F$ inside the block with number $(k, l, m)$ and $\boldsymbol{n}$ is an outward normal vector to the surface $S_{k, l, m}$.

Next, using Stokes' theorem we can rewrite (11b) in the integral form

$$
\int_{P_{1}}^{P_{2}} \frac{\boldsymbol{J} \cdot \boldsymbol{t}}{\kappa} d l=V\left(P_{1} ; \tau\right)-V\left(P_{2} ; \tau\right),
$$

where $P_{1}, P_{2}$ are points in $n^{3}$ and $t$ is the vector tangential to the curve of integration connecting these two points.

To compute the surface integral in formula (16) we assume that the current $\boldsymbol{J} \cdot \boldsymbol{n}$ is constant on each rectangular side of the block. To evaluate integrals of the type of (17), we also assume that $J \cdot t$ is constant on each line connecting two adjacent nodal points. This means that, for
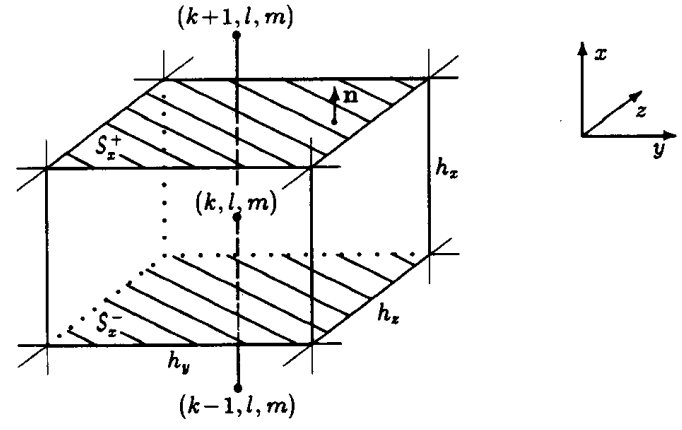

Fig. 2. Elementary block of the rectangular grid in the spatial domain.

example, on the line between the points $P_{k, l, m}$ and $P_{k+1, I, m}$ the current $\boldsymbol{J} \cdot \boldsymbol{t}$ is constant and equal to the current $\boldsymbol{J} \cdot \boldsymbol{n}$ on the surface $S_{x}^{+}$(see Fig. 2). This value of the current can be found from (17) as

$$
(\boldsymbol{J} \cdot \boldsymbol{n})_{\left.\right|_{\delta_{x}^{+}} ^{+}}=\frac{\kappa_{k+1, l, m}^{(x)}}{h_{x}}\left(\boldsymbol{V}_{k l, m}-V_{k+1, l, m}\right)
$$

where

$$
\kappa_{k+1, l, m}^{(x)}=\left(\frac{1}{h_{x}} \int_{P_{k, l, m}}^{P_{k+1, \ell, m}} \frac{1}{\kappa} d l\right)^{-1} .
$$

The coefficient $\kappa_{k+1, l, m}^{(x)}$ is called the effective admittance between the points $P_{k, l, m}$ and $P_{k+1, l, m}$. Similar expressions can be found for the normal component of the current $\boldsymbol{J}$ on other sides of the elementary block. Assuming that each elementary block contains only one type of tissue we arrive at the simple expressions for the effective admittances

$$
\left\{\begin{array}{l}
\kappa_{k, l, m}^{(x)}=2 \kappa_{k, l, m} \kappa_{k-1, l, m} /\left(\kappa_{k, l, m}+\kappa_{k-1, l, m}\right), \\
\kappa_{k, l, m}^{(y)}=2 \kappa_{k, l, m} \kappa_{k, l-1, m} /\left(\kappa_{k, l, m}+\kappa_{k, l-1, m}\right), \\
\kappa_{k, l, m}^{(z)}=2 \kappa_{k, l, m} \kappa_{k, l, m-1} /\left(\kappa_{k, l, m}+\kappa_{k, l, m-1}\right),
\end{array}\right.
$$

in which $\kappa_{k, f, m}$ denotes the value of $\kappa$ inside the block with number $(k, l, m)$. Assuming that the elementary block is not directly neighboring a boundary surface $\delta_{1}, \delta_{2}$, or $\Sigma$, we calculate the surface integral in (16) as

$$
\begin{aligned}
& \frac{1}{h_{x} h_{y} h_{z}} \int_{\delta_{k, l, m}} \int_{j} J \cdot n d s \\
& =\frac{1}{h_{x}^{2}}\left[\left(\kappa_{k, l, m}^{(x)}+\kappa_{k+1, l, m}^{(x)}\right) V_{k, l, m}-\kappa_{k, l, m}^{(x)} V_{k-1, l, m}\right. \\
& \left.\quad-\kappa_{k+1, l, m}^{(x)} V_{k+1, l, m}\right]+\frac{1}{h_{y}^{2}}\left[\kappa\left(\begin{array}{l}
(y) \\
k, l, m
\end{array}+\kappa_{k, l+1, m}^{(y)}\right) V_{k, l, m}\right. \\
& \left.\quad-\kappa_{k, l, m}^{(y)} V_{k, l-1, m}-\kappa_{k, l+1, m}^{(y)} V_{k, l+1, m}\right] \\
& \quad+\frac{1}{h_{z}^{2}}\left[\left(\kappa_{k, l, m}^{(z)}+\kappa_{k, l, m+1}^{(z)}\right) V_{k, l, m}-\kappa_{k, l, m}^{(z)} V_{k, l, m-1}\right. \\
& \left.\quad-\kappa_{k, l, m+1}^{(z)} V_{k, l, m+1}\right] .
\end{aligned}
$$


We can also describe boundary conditions (5) in terms of effective admittances. It is important to point out that the electrodes are modeled by blocks completely filled up with a metallic medium and with a prescribed potential in the nodal points of these blocks equal to $V_{q}$. Using the integral expression (18.b), the effective admittance $\kappa^{(x)}$ in a point adjacent to the electrode is arrived at

$\left\{\begin{aligned} \kappa_{k, l, m}^{(x)}= & 2 \kappa_{k-1, l, m}, \quad P_{k, l, m} \text { in electrode, } \\ & P_{k-1, l, m} \text { in tissue, } \\ \kappa_{k, l, m}^{(x)}= & 2 \kappa_{k, l, m}, \quad P_{k-1, l, m} \text { in electrode, } P_{k, l, m} \text { in tissue, }\end{aligned}\right.$

provided the admittance of metallic medium is equal to $\infty$. Similar formulas hold for the effective admittances $\kappa^{(y)}$ and $\kappa^{(z)}$

$\left\{\begin{aligned} \kappa_{k, l, m}^{(y)}= & 2 \kappa_{k, l-1, m}, \quad P_{k, l, m} \text { in electrode, } \\ & P_{k, l-1, m} \text { in tissue, } \\ \kappa_{k, l, m}^{(y)}= & 2 \kappa_{k, l, m}, \quad P_{k, l-1, m} \text { in electrode, } P_{k, l, m} \text { in tissue, }\end{aligned}\right.$

$\left\{\begin{aligned} & \kappa_{k, l, m}^{(z)}= 2 \kappa_{k, l, m-1}, \quad P_{k, l, m} \text { in electrode, } \\ & P_{k, l, m-1} \text { in tissue, } \\ & \kappa_{k, l, m}^{(z)}=2 \kappa_{k, l, m}, \quad P_{k, l, m-1} \text { in electrode, } P_{k, l, m} \text { in tissue. }\end{aligned}\right.$

To describe the impedance boundary conditions (9) in a similar way, we can assume that crossing the surface $\Sigma$ (see Fig. 1) the medium parameters do not change. Then, in the boundary nodal point the effective admittance $\kappa_{B}$ [cf. (19)] is equal to the admittance of the external medium. Examining (18.a) we observe that in order to calculate the current at the boundary surface $\Sigma$ we need to know the potential outside our grid as well. To avoid this problem we assume that the potential at the boundary surface $\Sigma$ is a mean value of the potentials $V_{\text {int }}$ and $V_{\text {ext }}$ on both sides of the surface; subsequently using the impedance boundary condition (9) we arrive at the relation

$$
\boldsymbol{J} \cdot \boldsymbol{n}_{\Sigma}=\frac{1}{2 \beta}\left(V_{\mathrm{int}}+V_{\mathrm{ext}}\right)
$$

On the other hand, from the (18.a) we have

$$
J \cdot n_{\Sigma}=\frac{\kappa_{B}}{h}\left(V_{\text {int }}-V_{\text {ext }}\right)
$$

where $\kappa_{B}$ is the admittance at the boundary $\Sigma$ and $h$ is the mesh size in either $x, y$, or $z$ direction. Eliminating the value of the exterior potential $V_{\text {ext }}$ from (24) and (25) we arrive at the relation

$$
\begin{aligned}
J \cdot n_{\Sigma} & =\frac{\kappa_{B}^{R}}{h} V_{\mathrm{int}}, \\
\kappa_{B}^{R} & =\frac{2 \kappa_{B} h}{2 \beta \kappa_{B}+h} .
\end{aligned}
$$

Now we can evaluate the surface integral using (20) also in the points adjacent to the surface $\Sigma$ assuming that in each point outside this surface the electric potential $V_{\text {ext }}$ is equal to zero and an effective admittance at that point is calculated through (26.b).

\section{Finite Difference Approximation in $\tau$ COORDinate}

In order to obtain the computational iterative scheme we apply some finite difference formula for the $\tau$ derivative in the left-hand side of (16). For this purpose we introduce the discretization of the $\tau$ coordinate as follows

$$
\begin{aligned}
& \tau_{0}, \tau_{1}, \tau_{2}, \cdots, \tau_{n}, \tau_{n+1}, \cdots, \\
& \quad \text { where } \tau_{0}<\tau_{1}<\tau_{2}<\cdots<\tau_{n}<\tau_{n+1}<\cdots,
\end{aligned}
$$

and the notation $V^{(n)}, J^{(n)}$ for electric potential $V$ and current $J$ evaluated at $\tau=\tau_{n}$ as

$$
\begin{aligned}
& V^{(n)}=V\left(x, y, z ; \tau_{n}\right), \\
& J^{(n)}=\boldsymbol{J}\left(x, y, z ; \tau_{n}\right) .
\end{aligned}
$$

Now, let us apply for the $\tau$ derivative appearing on the left-hand side of (16) the leap-frog formula

$$
\begin{aligned}
\left(\frac{\partial V}{\partial \tau}\right)_{\mid \tau=\tau_{n}} & =\frac{V^{(n+1)}-V^{(n-1)}}{\tau_{n+1}-\tau_{n-1}} \\
& =\frac{1}{\delta \tau_{n}+\delta \tau_{n+1}}\left(V^{(n+1)}-V^{(n-1)}\right)
\end{aligned}
$$

where $\delta \tau_{n}$ is the size of the ( $n$ )th $\tau$ step along the iterative coordinate, $\delta \tau_{n}={ }_{\text {def }} \tau_{n}-\tau_{n-1}$. Using Taylor's series expansion [4], we can find the exact equivalence of our leapfrog formula (29) as

$$
\begin{aligned}
& \frac{1}{\delta \tau_{n}+\delta \tau_{n+1}}\left(V^{(n+1)}-V^{(n-1)}\right) \\
& =\left(\frac{\partial V}{\partial \tau}+\frac{\delta \tau_{n+1}-\delta \tau_{n}}{2} \frac{\partial^{2} V}{\partial \tau^{2}}\right. \\
& \left.\quad+\frac{1}{6} \frac{\left(\delta \tau_{n+1}\right)^{3}+\left(\delta \tau_{n}\right)^{3}}{\delta \tau_{n+1}+\delta \tau_{n}} \frac{\partial^{3} V}{\partial \tau^{3}}+\cdots\right)_{\mid \tau=\tau_{n}} .
\end{aligned}
$$

From (30) we observe that in the case of equal steps $\delta \tau_{n}$ $=\delta \tau_{n+1}$ the formula (29) has second-order accuracy and almost second-order accuracy is achieved providing $\delta \tau_{n+1}$ $-\delta \tau_{n}$ is relatively small.

Using (29) in (16) we arrive at the iterative scheme

$$
V_{k, l, m}^{(n+1)}=V_{k, l, m}^{(n-1)}-\frac{\delta_{n} F_{k, l, m}}{h_{x} h_{y} h_{z}} \iint_{\delta_{k, l, m}} J^{(n)} \cdot \boldsymbol{n} d s
$$

where the integral over the surface $\delta_{k, l, m}$ is evaluated using (20) with $V=V^{(n)}$. In (31), $\delta_{n}$ is defined as $\delta_{n}={ }_{\text {def }} \delta \tau_{n}$ $+\delta \tau_{n+1}=\tau_{n+1}-\tau_{n-1}$.

Although, for equal $\tau$ steps, (31) implies second-order accuracy with respect to the $\tau$ discretization, this scheme 
is numerically unstable [3]. It means that when we start our iterative process with two properly chosen fields $V^{(0)}$ and $V^{(1)}$, after some number of iteration steps a small inaccuracy together with round-off errors cause the process to start to diverge.

To eliminate the instability of the scheme of (31) we apply an averaging procedure

$$
V_{k, l, m}^{(n)}=\theta V_{k, l, m}^{(n+1)}+(1-\theta) V_{k, I, m}^{(n-1)}
$$

where the weight factor $\theta$ is the real number satisfying $1 / 2 \leq \theta \leq 1$. Averaging with $\theta=1 / 2$ is used in the derivation of the stable Dufort-Frankel scheme for the heat-diffusion equation [3]. In the case of convergence the $(n)$ th solution $V^{(n)}$ may be closer to $V^{(n+1)}$ than to $V^{(n-1)}$; then, the averaging formula (32) gives better results with $\theta>1 / 2$ than with $\theta=1 / 2$.

Applying the averaging formula of (32) to the scheme of (31) the final iterative scheme is arrived at

$$
V_{P}^{(n+1)}=\frac{\delta_{n}\left(\Lambda V^{(n)}\right)_{P}}{1+\theta \delta_{n} \Lambda_{P}}+\frac{1-(1-\theta) \delta_{n} \Lambda_{P}}{1+\theta \delta_{n} \Lambda_{P}} V_{P}^{(n-1)},
$$

where $P=P_{k, l, m}$ is the point of the mesh, $\boldsymbol{\Lambda}$ is the spatial difference operator of the form

$$
\begin{aligned}
(\boldsymbol{\Lambda} V)_{P_{k, l, m}}= & \frac{F_{k, l, m}}{h_{x}^{2}}\left(\kappa_{k, l, m}^{(x)} V_{k-1, l, m}+\kappa_{k+1, l, m}^{(x)} V_{k+1, l, m}\right) \\
& +\frac{F_{k, l, m}}{h_{y}^{2}}\left(\kappa_{k, l, m}^{(y)} V_{k, l-1, m}+\kappa_{k, l+1, m}^{(y)} V_{k, l+1, m}\right) \\
& +\frac{F_{k, l, m}}{h_{z}^{2}}\left(\kappa_{k, l, m}^{(z)} V_{k, l, m-1}\right. \\
& \left.+\kappa_{k, l, m+1}^{(z)} V_{k, l, m+1}\right),
\end{aligned}
$$

and $\Lambda_{P}$ is the complex number given by

$$
\begin{aligned}
\Lambda_{P_{k, l, m}}= & F_{k, l, m}\left(\frac{\kappa_{k, l, m}^{(x)}+\kappa_{k+1, l, m}^{(x)}}{h_{x}^{2}}\right. \\
& \left.+\frac{\kappa_{k, l, m}^{(y)}+\kappa_{k, l+1, m}^{(y)}}{h_{y}^{2}}+\frac{\kappa_{k, l, m}^{(z)}+\kappa_{k, l, m+1}^{(z)}}{h_{z}^{2}}\right) .
\end{aligned}
$$

Equations (33)-(35) are valid at all the mesh points $P_{k, l, m}$ when the formulas for effective admittances of Section IV are used.

\section{Numerical Implementation}

To obtain numerical results for the volume density of dissipated EM power, this quantity is calculated using (4). It is important to notice that in Section IV we have assumed that the current is constant on the line connecting two adjacent nodal points and equal to the value of the normal component of the current on the block-surface perpendicular to this line between the two nodal points. This means that the current flowing between the points
$P_{k-1.1, m}$ and $P_{k, I, m}$ is equal to the value of $(\boldsymbol{J} \cdot \boldsymbol{n})_{\mid \delta_{\bar{r}}}$ and the current flowing between the points $P_{k, 1, m}$ and $P_{k+1,1, m}$ is equal to the $(\boldsymbol{J} \cdot \boldsymbol{n})_{\mid \mathrm{s}_{\mathrm{r}}^{+}}$, see Fig. 2. Using the second expression of (4) we can find the EM power dissipated by $x$ component of generalized current in the block $(k, l, m)$ as

$$
\dot{w}_{x: k, I, m}=\frac{\sigma_{k, I, m}}{4\left|\kappa_{k, l, m}\right|^{2}}\left[\left|J_{x: k, l m}^{+}\right|^{2}+\left|J_{x ; k, I, m}^{-}\right|^{2}\right] .
$$

where the superscripts denote the values of the $x$ component of $J$ at the left-hand " _. " and the right-hand side " + " of the block. Using (18a) we can find these currents as

$$
\begin{aligned}
& J_{x ; k, l, m}^{-}=\frac{\kappa_{k, l, m}^{(x)}}{h_{x}}\left(V_{k-1, l, m}-V_{k, l, m}\right), \\
& J_{x ; k, l, m}^{+}=\frac{\kappa_{k+1, l, m}^{(x)}}{h_{x}}\left(V_{k, l, m}-V_{k+1, l, m}\right) .
\end{aligned}
$$

In a similar way we can find the $y$ and $z$ components of the current $J$ and complete the calculation of the EM power dissipated in a particular block.

To satisfy Kirchhoff's condition (8) we need to find the proper values of the potentials $V_{1}$ and $V_{2}$ on both electrodes satisfying (7). This can be done by starting with the initial values of (13c) which are the exact potentials for the symmetric configuration. During the iterative process, after each $m$ iterations, we need to correct the potentials on the electrodes to enforced Kirchhoff's law (8). This can simple be carried as follows:

$$
\begin{aligned}
& V_{q}^{(n+m)}=\frac{U Z_{q}^{(n+m)}}{Z_{1}^{(n+m)}+Z_{2}^{(n+m)}}, \\
& Z_{q}^{(n+m)}=\frac{V_{q}^{(n)}}{I_{q}^{(n+m)}}, \quad q=1,2,
\end{aligned}
$$

where the current $I_{q}^{(n+m)}$ is the current after $n+m$ iterations flowing out from $q$ electrode with the potential $V_{q}^{(n)}$. These currents are evaluated using (6), (18a), and (21)-(23).

To start the averaging-leap-frog iterative scheme (ALF) described in Section $V$ we need to know two starting values of the electric potentials $V^{(0)}$ and $V^{(1)}$ in all mesh points and the size $\delta \tau_{1}$ of the first $\tau$ step. One of the simplest possibilities, which proves to be very efficient in practice, is to start the ALF iterative scheme with

$$
\left\{\begin{array}{l}
V^{(0)}=V^{(1)} \equiv 0 \text { in all grid points, } \\
\delta \tau_{1}=0 .
\end{array}\right.
$$

The potential $V^{(2)}$, obtained from (33) with initialization (39) and with some $\delta \tau_{2}>0$, will have nonzero values at the points adjacent to the electrode surfaces and zero values at other grid points. When the scheme continues the electric potential $V^{(n)}$ will penetrate through the configuration ("diffusion") until the equilibrium situation is arrived at. 
As a measure of the convergence, the normalized integrated square error with respect to the satisfaction of the original three-dimensional problem is introduced to

$$
\begin{aligned}
\operatorname{ERR}_{V}^{(n)}= & \left(\sum_{k, l, m}\left|\iint_{\delta_{k, l, I, m}} \boldsymbol{J}^{(n)} \cdot \boldsymbol{n} d s\right|^{2}\right)^{1 / 2} / \\
& \left(\sum_{k, l, m}\left|\iint_{s_{k, l, m}} \boldsymbol{J}^{(n)} \cdot \boldsymbol{n} d s\right|^{2}\right)^{1 / 2}
\end{aligned}
$$

where the surface integrals are evaluated using (20). In the case when the initialization of (39) is used, the denominator in (40) is equal to the value of the integral of the normal component of the initial current $J^{(0)}$ over the electrode surfaces. As a second measure the normalized power balance error is introduced as

$$
\operatorname{ERR}_{P}^{(n)}=\frac{P_{i}^{(n)}-P_{d}^{(n)}-P_{r}^{(n)}}{P_{i}^{(n)}},
$$

where $P_{i}^{(n)}$ is the input power fed to the electrodes

$$
P_{i}^{(n)}=\frac{1}{2} \operatorname{Re}\left[V_{1}^{(n)} I_{1}^{(n)}+V_{2}^{(n)} I_{2}^{(n)}\right]
$$

$P_{d}^{(n)}$ is the power dissipated in the volume $V_{\Sigma}$ of the whole applicator configuration enclosed within the surface $\Sigma$,

$$
P_{d}^{(n)}=\iiint_{V_{\Sigma}} \dot{w}^{(n)} d v
$$

and $P_{r}^{(n)}$ is the power flowing out through the surface $\Sigma$,

$$
P_{r}^{(n)}=\frac{1}{2} \iint_{\Sigma} \operatorname{Re}\left[V_{\mid \Sigma}^{(n)}\left(J^{(n)} \cdot n_{\Sigma}\right)^{*}\right] d s .
$$

The volume density of dissipated EM power $\dot{w}$ in (43) is evaluated using (4), (36), and (37), the current $\boldsymbol{J}^{(n)} \cdot \boldsymbol{n}_{\Sigma}$ in (44) is calculated from (26a) and the potential $V_{\mid \Sigma}^{(n)}$ is found from the impedance boundary condition (9) after the current $\boldsymbol{J}^{(n)} \cdot \boldsymbol{n}_{\Sigma}$ is known.

After some numerical experiments we have observed that the speed of convergence of the ALF method depends on the choice of the specific impedance $\beta$ introduced in (9). The most rapid convergence was achieved for $\beta=0$. In this case the external boundary surface $\Sigma$ becomes the third electrode with the electric potential equal to 0 . However, to obtain realistic results in this case, the truncation distance between the applicator and $\Sigma$ should be significantly enlarged at the cost of an increase in the number of grid elements and this is not recommended in practice. From this point of view the Neumann boundary condition at $\Sigma, \beta=\infty$, which is equivalent to the $\boldsymbol{J} \cdot \boldsymbol{n}_{\Sigma}=0$, allows us to work with the minimum number of grid elements by locating surface $\Sigma$ very close to the applicator. Unfortunately, in this case the convergence was much slower than the one for $\beta=0$. The best situation can be achieved with some finite value of $\beta$ and the number of grid elements slightly larger than if $\beta=\infty$. To be able to deal with a nonzero and finite value of $\beta$ we have established some physical meaning of this parameter as follows. Let us assume that the potential $V$ decays outside our grid linearly and reaches the value 0 within a distance $d$ in the direction of outward normal $n_{\Sigma}$ from the surface $\Sigma$. With these assumptions the normal derivative of $V$ at the surface $\Sigma$ is equal to $-d^{-1}$. Now, using (3b) and (9), we can find the value of $\beta$ as

$$
\beta=\frac{d}{\kappa_{B}}
$$

The physical meaning of this particular value of the specific surface impedance $\beta$ is such that the whole configuration shown in Fig. 1 is continuously extended in each direction, and next enclosed in a Faraday box at a distance $d$ from the surface $\Sigma$ with zero electric potential.

We finally consider the spatially dependent relaxation function $F$. Very large differences in the admittance $\kappa$ in the different tissues and the fact that all these values are complex numbers reduce the convergence rate of our scheme dramatically. To accelerate the convergence, extensive numerical experiments have shown that a suitable choice for this function appears to be

$$
F_{k, l, m}=\left(\kappa_{k, l, m}\right)^{-(1 / 2)} \frac{\min \left(h_{x}, h_{y}, h_{z}\right)}{\max (|\kappa|)}
$$

where $\max (|\kappa|)$ denotes the maximum value of $\left|\kappa_{k, l . m}\right|$ in the configuration. The second factor in (46) is added for normalization purposes, while the first, spatially dependent factor, reduces large differences of the values of $(\boldsymbol{\Lambda} V)_{P_{k, l, m}}$ and $\Lambda_{P_{k, l, m}}$ in different points of the grid. This choice of relaxation function $F$ overcomes the decrease of convergence and facilitates an iterative solution for problems where the quantity $\max (|\kappa|) / \min (|\kappa|)$ exceeds the value of 1000 and where the complex value of $\kappa$ in one of the media of our model, namely air, is almost imaginary.

The configuration of the model used in the numerical program is characterized in a three-dimensional integer array; each integer represents the type of the tissue or the presence of an electrode in the current block of the mesh. To save computational time in the evaluation of the expressions appearing in (19), the effective admittance between the points located in two different tissues is computed only once and stored in a two-dimensional complex array $N_{T} \times N_{T}$ where $N_{T}$ is the number of different tissues used in the computer model.

\section{Numerical Results}

\section{A. Test Example}

As a test example we chose the radially layered model of a human thigh. This is a three-dimensional model in Cartesian coordinates system while in a polar cylindrical coordinates system the field quantities depends only on the axial and radial coordiantes. Using this rotational symmetry of the field and of the test model, the EM power distribution in the human thigh have been computed previously by an integral equation method [2]. 
At the external boundary surface $\Sigma$ the specific impedance $\beta$ appearing in the impedance boundary condition (9) was calculated from (45) where the distance $d$ was equal to $5 \mathrm{~m}$ in the longitudinal $z$ direction and to $0.5 \mathrm{~m}$ in $x$ and $y$ direction. This choice enforced the outflow of energy through the surface $\Sigma$ to a negligibly small value.

The cross section of the human thigh was modeled into a $35 \times 35$ square array with $h_{x}=h_{y}=3.72 \mathrm{~mm}$. The ring electrodes and the air gap between the electrode and the thigh model were replaced by a circular construction of single blocks with air and metal blocks, respectively. In this way, the cross section of human thigh + air-gap + electrode was modeled in a $39 \times 39$ array. The cross section of the whole configuration shown in Fig. 1 was described by a $51 \times 51$ array with six rows and six columns added to each side of the thigh + air-gap + electrode array. Finally, the whole three-dimensional configuration was modeled into $51 \times 51 \times 45=117045$ blocks where the length in the $z$ direction of each block was taken as $h_{z}=8 \mathrm{~mm}$. Both ring-shaped electrodes were $32 \mathrm{~mm}$ wide and were located with $200 \mathrm{~mm}$ distance between them. The electromagnetic parameters and the outer diameters of the tissue layers used in the computer model of the human thigh are listed in Table I.

We performed some experiments with the choice of the $\tau$ step $\delta \tau_{n}$ and the weight factor $\theta$ examining the speed of convergence of the method. A strong dependence on the $\delta \tau_{n}$ has been found. It was also found that a suitable improvement in convergence is achieved when $\delta \tau_{n}$ is growing at the beginning of the iterative procedure and, after a few hundreds iterations, $\delta \tau_{n}$ is slightly decaying. The choice of the weight factor $\theta$ has been found less critical provided that $1 / 2 \leq \theta \leq 1$. Taking $\theta<1 / 2$ we have detected that the scheme is divergent in most cases. For the test model described above the most efficient procedure was found to be the following one: the relaxation function $F$ defined by (46) was used with the $\tau$ step $\delta \tau_{n}$ growing sinusoidally from $\delta \tau_{2}=4$ to $\delta \tau_{300}=15$ and next decreasing to $\delta \tau_{420}=10$. The weight factor $\theta$ was decreased from $\theta=0.53$ to $\theta=0.5$ in the first 40 iterations and next was kept constant with value $\theta=0.5$ up to the moment, when after 320 iterations $\delta \tau_{n}$ stopped its growth. In the last 100 iterations, $\theta$ was increased again to the value $\theta=0.51$. Iterating in this way, after 280 iterations the normalized integrated square error defined by (40) was equal to $\operatorname{ERR}_{V}^{(280)}=0.24 \%$ when the normalized power balance error defined by (41) was equal to $\operatorname{ERR}_{P}^{(280)}=$ $2.3 \%$. After 420 iterations both errors were decreased to $\operatorname{ERR}_{V}^{(420)}=0.05 \%$ and $\operatorname{ERR}_{P}^{(420)}=0.04 \%$. Further iterations did not improve the $\mathrm{ERR}_{V}$ error decreasing only the power balance error to $\mathrm{ERR}_{P}^{(\infty)} \approx 0.01 \%$ which seems to be the maximum accuracy which can be achieved in the single-precision arithmetic used by the computer. The computer program was written in Fortran. The CPU computation time of one iteration on a VAX-8250 computer amounts to $80 \mathrm{~s}$.

For the human thigh model, the relative EM power distribution computed by our ALF iterative method and by
TABLE 1

Parameters of the Radially Layered Thigh Model Used iN Computer Program $(f=27.12 \mathrm{MHz})$

\begin{tabular}{lcrllll}
\hline \hline \multicolumn{2}{c}{ Medium } & & & & \multicolumn{2}{c}{$\begin{array}{c}\text { Admittance } \\
\kappa=j \omega \epsilon+\sigma\end{array}$} \\
\cline { 6 - 7 } Name & $\begin{array}{c}\text { Out Diam. } \\
{[\mathrm{mm}]}\end{array}$ & $\begin{array}{c}\text { Relative } \\
\text { Permittivity }\end{array}$ & \multicolumn{1}{c}{$\begin{array}{c}\text { Conductivity } \\
\sigma[\mathrm{S} / \mathrm{m}]\end{array}$} & Real $(\kappa)$ & Imag $(\kappa)$ \\
\hline Marrow & 11.2 & 30.0 & 0.46 & 0.46 & 0.0453 \\
Bone & 41.0 & 7.3 & 0.028 & 0.028 & 0.011 \\
Muscle & 108.0 & 113.0 & 0.61 & 0.61 & 0.17 \\
Fat & 123.0 & 20.0 & 0.047 & 0.047 & 0.0302 \\
Skin & 130.5 & 113.0 & 0.61 & 0.61 & 0.17 \\
& Air & 1.0 & 0.0000001 & 0.0000001 & 0.0015 \\
\hline
\end{tabular}

the integral equation method (IEM) [2], is shown in Fig. 3 . In both patterns the EM power distribution is normalized to the average dissipated EM power in the tissue within the domain between the rings. In the marrow, bone, and muscle tissues we observe an excellent agreement between both patterns. Some discrepancies appear in the fat and skin tissues where higher EM power deposition is predicted by the ALF method. In the author's opinion, these higher values are mainly caused by the block-shaped surfaces of the thigh cylinder and ring electrodes in the ALF method which results in the grid model in a larger effective surface of the electrodes. The input capacitance of the whole applicator computed for the grid configuration was about $12 \%$ higher than the capacitance found for the circular model used in IEM. This fact can result in a higher value of the electric field in the layers adjacent to the air-gap in the same ratio, and accordingly to (4), in an about $25 \%$ higher value of dissipated EM power in those layers. This phenomenon is visible in the skin layer but also in the fat layer where in the neighborhood of the electrodes hot spots are produced by the radial electric field [1], [2]. On the other hand, the averaging differentiation in radial direction used in IEM can have a negative influence on the prediction of the hot-spot values computed by IEM. In this way, the discrepancies between presented patterns can be explained. These discrepancies should be significantly decreased when the model with larger air-gap is cmputed by the ALF iterative method.

\section{B. Knee Model}

After obtaining positive results with the test model of a human thigh, the knee joint of the left leg with the parts of thigh and mid-calf were modeled into $41 \times 41 \times 36$ $=60516$ blocks. To build this model, the CT images of the supine left leg of a 25 -year-old woman were used. The model was designed with the help of the Dr. D. Den Hoed Kliniek in Rotterdam, The Netherlands, where these CT images were taken.

At first, 20 CT images with a $10 \mathrm{~mm}$ scanning interval were converted into 20 two-dimensional tissue type arrays with the aid of the gray scale of each image. The number of pixels on each CT image was reduced by the factor of $9,(3 \times 3)$, to obtain a $41 \times 41$ array. The Houndsfield 


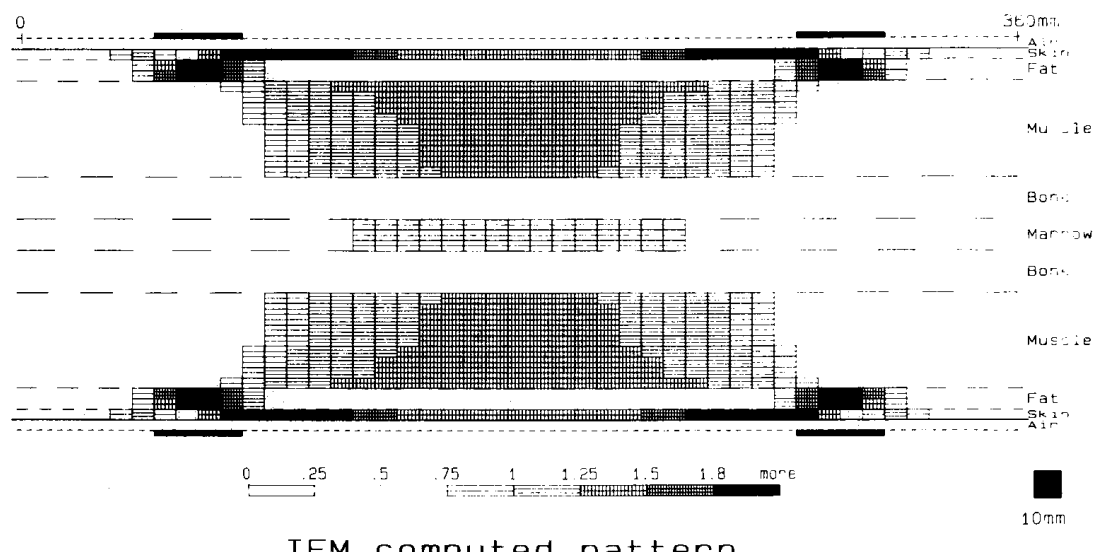

IEM computed pattern

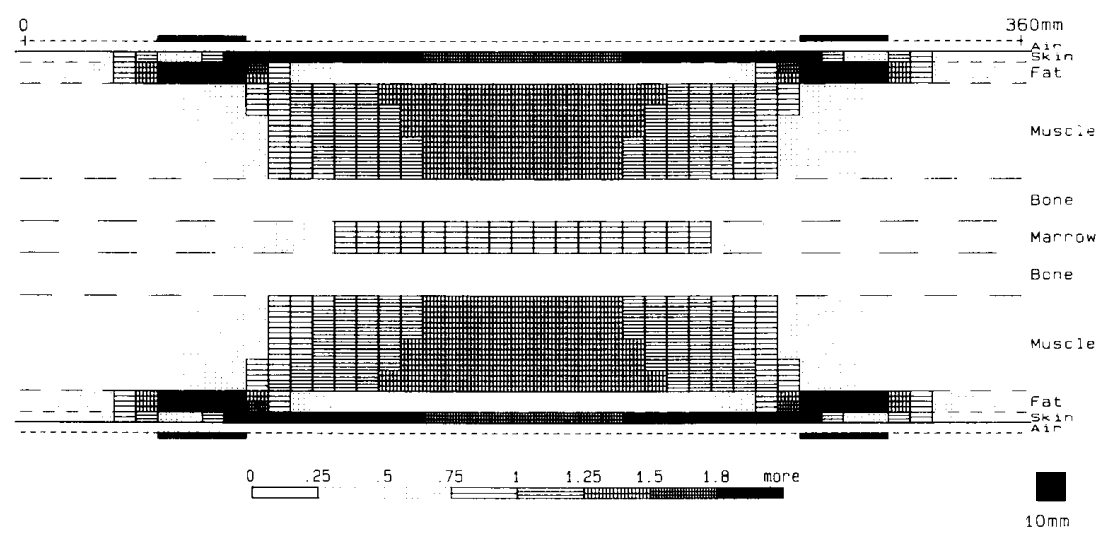

ALF computed pattern

Fig. 3. The EM power distribution patterns for the model of the human thigh computed by the ALF and IEM methods; air gap $=3.72 \mathrm{~mm}$, electrode width $=32 \mathrm{~mm}$, distance between electrodes $=200 \mathrm{~mm}$, fre quency $=27.12 \mathrm{MHz}$.

numbers of each CT image were automatically converted into a tissue types, namely, muscle, fat, or bone. In the next step, these tissue type images were compared to the original images by a professional radiologist and adjusted for improper tissue allocation. Furthermore, another adjustment regarding marrow, cartilage, nerve, etc., were made with the Cross-Sectional Anatomy Atlas [5] and pictures found in [6]. The model thus obtained consists of 20 slices, each of $10 \mathrm{~mm}$ in thickness. Finally, to extend this model with some truncation distance from the electrodes, the first and the last tissue type image have been duplicated eight times forming a continuous part of the thigh and mid-calf at each end of the model.

When the $41 \times 41 \times 36$ block model of the knee was completed, the pair of $30 \mathrm{~mm}$ wide electrodes with an internal diameter $135 \mathrm{~mm}$ was added to the model. The electrodes were located with $200 \mathrm{~mm}$ distance between them and adjusted in such a way that the air-gap between the first electrode and the thigh surface varies from 6 to $12 \mathrm{~mm}$ and between the second electrode and the mid-calf surface varies from 12 to $21 \mathrm{~mm}$. The distance between the external surfaces of the electrodes and the boundary surface $\Sigma$ varies from 12 to $18 \mathrm{~mm}$. The whole model of the knee joint with part of the thigh, part of the mid-calf, with the pair of electrodes and with some space around the applicator was build with $57 \times 57 \times 36=116964$ homogeneous blocks. The electromagnetic parameters used for the knee model are listed in Table II.

At the external boundary surface $\Sigma$, as before, the specific impedance $\beta$ was calculated from (45). Now, the distance $d$ was equal to $0.8 \mathrm{~m}$ in the longitudinal $z$ direction and to $0.15 \mathrm{~m}$ in $x$ and $y$ direction. With this choice of $\beta$ the power $P_{r}$ flowing out through the surface $\Sigma$ [cf. (44)] was found as $16 \%$ of the input power $P_{i}$, [cf. (42)].

During the iterations, the relaxation function defined by (46) was used. The $\tau$ step $\delta \tau_{n}$ was grown sinusoidally from $\delta \tau_{2}=4$ to $\delta \tau_{450}=16$ and next, sinusoidally varying between 8 and 16 with the period equal to 300 iterations. The weight factor $\theta$ was parabolically decreased from 0.54 to 0.5 within first 50 iterations, and then only slightly var- 
TABLE II

Parameters Used in Computer Program for the Model of the Knee JolNT $(f=27.12 \mathrm{MHz})$

\begin{tabular}{lcl}
\hline \hline Medium & $\begin{array}{c}\text { Relative } \\
\text { Permittivity } \\
\epsilon\end{array}$ & $\begin{array}{c}\text { Conductivity } \\
\sigma[\mathrm{S} / \mathrm{m}]\end{array}$ \\
\hline Air & 1.0 & 0.0000001 \\
Marrow & 30.0 & 0.46 \\
Bone & 7.3 & 0.028 \\
Fat & 20.0 & 0.047 \\
Muscle & 113.0 & 0.61 \\
Skin & 102.0 & 0.5 \\
Ligament* & 17.0 & 0.15 \\
Blood & 118.0 & 0.8 \\
Nerve & 150.0 & 0.25 \\
Cartilage* & 12.0 & 0.09 \\
\hline
\end{tabular}

*Data estimated from the water content.

ied within the range $\theta \in(0.498,0.505)$. After each 25 iterations the potentials on the electrodes were corrected according to the relations of (38) and then the weight factor $\theta$ was slightly increased with the growth proportional to the change of the electrode potentials. Both measures of the convergence introduced by (40) and (41) were used. The resulting errors are presented in Table III.

During the first 200 iterations the potential field $V$ was penetrating to all the bricks in the configuration, starting from the electrodes. Quite realistic values of $V$ were established in the neighborhood of the electrodes while near the external surface $\Sigma$ the values of $V$ were still far away from the final solution. This resulted in the fast decrease of $\mathrm{ERR}_{V}$, but the erroneous solution near the external boundary $\Sigma$ led to nonsatisfaction of the power balance. The next 250 iterations significantly improved our solution near the boundary surface and gave the first approximate value of the outflowing power $P_{r}$. In the next 300 iterations the results were improved again. After 750 iterations we obtained the first results yielding realistic patterns of the EM power distribution in our model. Continuing this process we still observed some oscillating behavior of the convergence which resulted in irregular decreasing of the error measures $\mathrm{ERR}_{V}$ and $\mathrm{ERR}_{P}$. The complex potentials satisfying (8) with $U=2 \mathrm{~V}$ were found as: $V_{1}=(0.85,0.02) \mathrm{V}$ for the electrode located near the thigh and $V_{2}=-(1.15,0.02) \mathrm{V}$ for the electrode located near the mid-calf.

One more numerical experiment was done to check whether the choice of the specific surface impedance $\beta$ of (45) is the proper one. The thigh part of the knee model was continuously extended in $z$ direction by adding five more thigh cross sections. Then, the value of $\beta$ used at the thigh part of the boundary surface $\Sigma$ was computed from (45) with $d=0.75 \mathrm{~m}$ instead of $0.8 \mathrm{~m}$ used before. The results, for the extended $57 \times 57 \times 41=133209$ block model, show no large differences from the results of the nonextended model. The only significant difference found for the extended model was the decrease of the outflowing power, viz., $P_{r}=15.83 \%$. The difference from the former results is equal to $0.73 \%$ of the input power.
TABLE III

Convergence of Alf Iterative Process in the Case of the Knfe MODEL

\begin{tabular}{cccc}
\hline & \multicolumn{2}{c}{ Errors } & \\
\cline { 2 - 3 } $\begin{array}{c}\text { Number of } \\
\text { Iterations }\end{array}$ & $\begin{array}{c}\mathrm{ERR}_{\psi} \\
\text { from (40) }\end{array}$ & $\begin{array}{c}\text { ERR }_{P} \\
\text { from (41) }\end{array}$ & $\begin{array}{c}\text { Outflowing } \\
\text { Power } \boldsymbol{P}_{r} \\
\text { from (44) }\end{array}$ \\
\hline 200 & $1.26 \%$ & $43.23 \%$ & $4.43 \%$ \\
450 & $0.68 \%$ & $10.16 \%$ & $14.14 \%$ \\
750 & $0.49 \%$ & $1.60 \%$ & $16.11 \%$ \\
1350 & $0.26 \%$ & $0.24 \%$ & $16.48 \%$ \\
1950 & $0.13 \%$ & $0.16 \%$ & $16.53 \%$ \\
2550 & $0.06 \%$ & $0.13 \%$ & $16.56 \%$ \\
\hline
\end{tabular}

This difference can be explained by the extra power dissipation in the extended part of the thigh.

The results obtained for the knee model are presented in some cross sections: one sagittal cross section $[Z X(1)]$ is shown in Fig. 4(a), one coronal cross-section [ $Z Y(1)]$ is shown in Fig. 4(b), and four transverse cross sections $[X Y(1)-X Y(4)]$ are shown in Fig. 4(c)-(f). On the top of each of these figures we present the tissue configuration in the current cross section with other cross sections marked by the solid lines. The EM power distribution patterns, normalized to the average dissipated power in the tissue domain between the rings, are shown below. All the results shown in Figs. 4(a)-(f) were obtained after 2550 iterations and they have been compared with the results obtained after 1350 iterations. No visible difference was found when examining the normalized EM power distribution patterns.

The main phenomenon that can be observed from the presented patterns is extremely high EM power deposition in the muscle tissue at the back of the knee joint, see Fig. 4(b). In the previous case of the cylindrically layered test model (Fig. 3), the maximum amount of the EM power distribution occurred in the fat tissue located close to the electrodes or in the skin. This maximum was approximately two times higher than the maximum magnitude of the EM power distributed in the muscle tissue. In the case of the knee model, the most heated tissues were the sartorius and biceps femoris muscles in the parts close to the joint. Also the semimembranous and the heads of the gastrocnemius muscle were treated quite heavily, while in the quadriceps femoris the level of EM power deposition was about three times lower than in the hamstrings, see Fig. 4(c)-(f). A low level of EM power deposition was also observed in the bone-marrow tissue as well as in the other tissues of the knee joint.

\section{CONCLUSION}

We have developed an efficient iterative scheme to solve three-dimensional quasi-static electromagnetic field problems with strongly inhomogeneous media. The number of iterations amounts to about 1000 for our present problem with 120000 unknowns. Double precision arithmetic are not needed in the case of a required accuracy of about $1 \%$. 


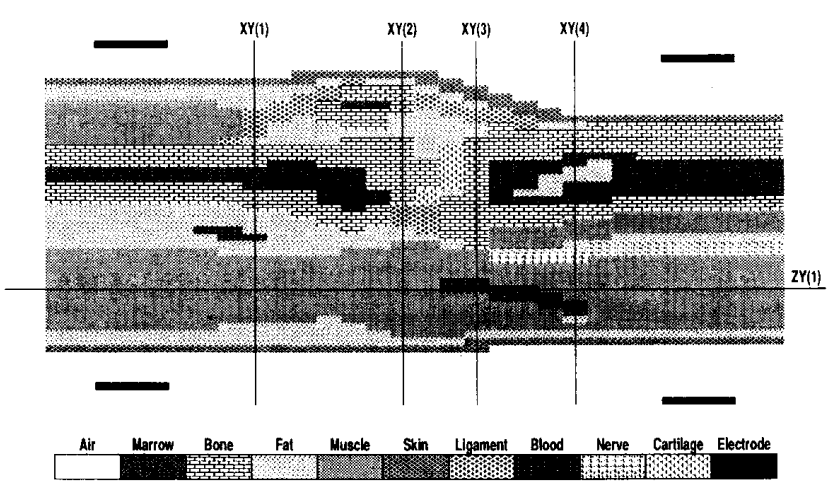

Tissue in ZX(1) cross-section

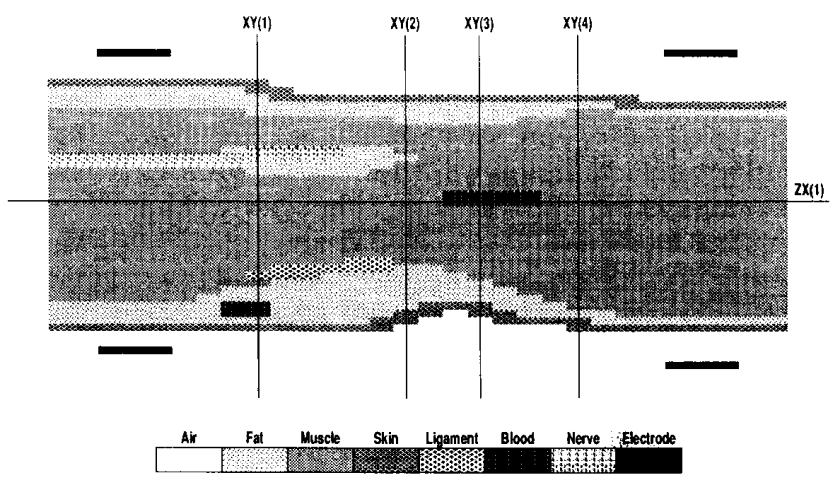

Tissue in ZY(1) cross-section

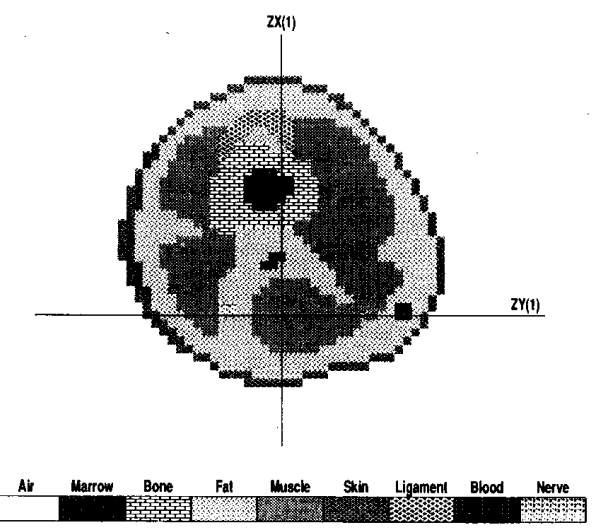

Tissue in $\mathrm{XY}(1)$ cross-section

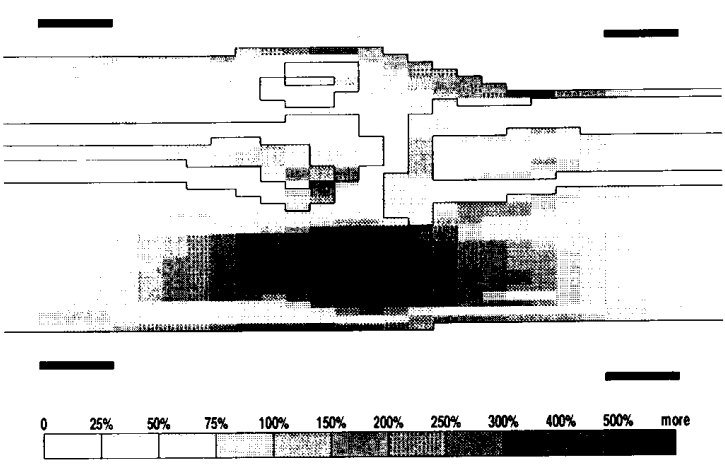

EM power distribution in ZX(1) cross-section

$2 \times(11)$

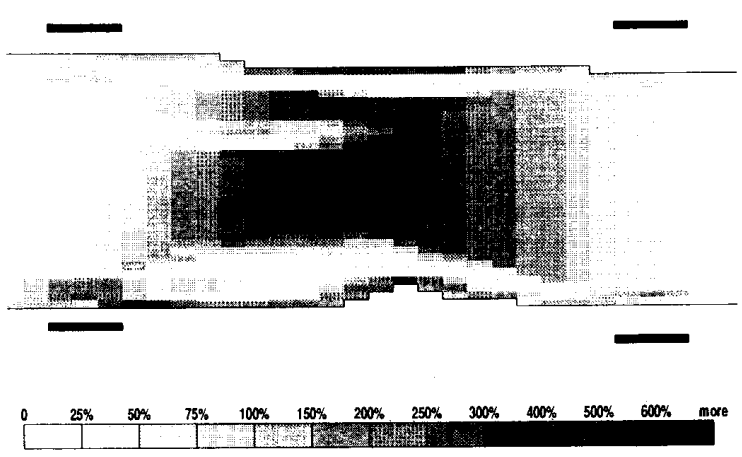

EM power distribution in ZY(1) cross-section (b)

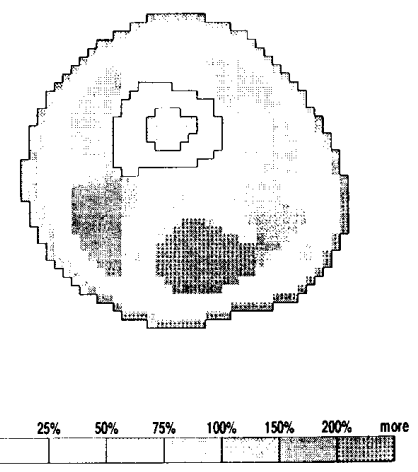

EM power distribution in $\mathrm{XY}(1)$ cross-section

Fig. 4. (a) Central cross section of the knee model in the sagittal plane $Z X(1)$ and the EM power distribution in $Z X(1)$ computed by the ALF method. (b) Cross section of the back part of the knee model in the coronal plane $Z Y(1)$ and the EM power distribution in $Z Y(1)$ computed by the ALF method. (c) Cross section of the lower part of thigh of the knee model in the transverse plane $X Y(1)$ and the EM power distribution in $X Y(1)$ computed by the ALF method. (d) Cross section of the upper part of knee joint of the knee model in the transverse plane $X Y(2)$ and the EM power distribution in $X Y(2)$ computed by the ALF method. (e) Cross section of the lower part of knee joint of the knee model in the transverse plane $X Y(3)$ and the EM power distribution in $X Y(3)$ computed by the ALF method. (f) Cross section of the lower part of mid-calf of the knee model in the transverse plane $X Y(4)$ and the EM power distribution in $X Y(4)$ computed by the ALF method. 


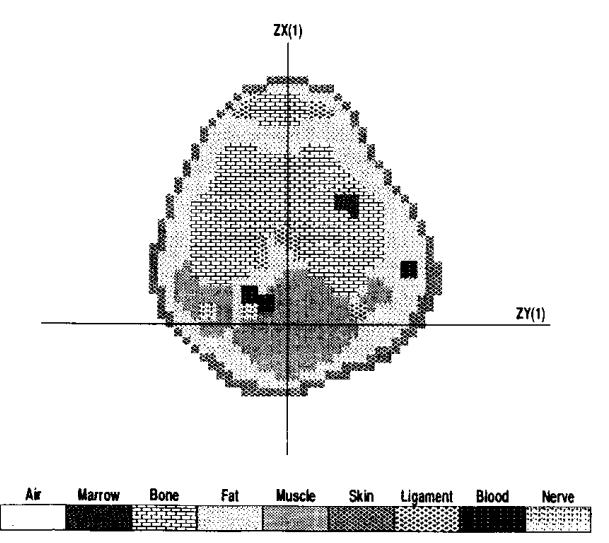

Tissue in $\mathrm{XY}(2)$ cross-section
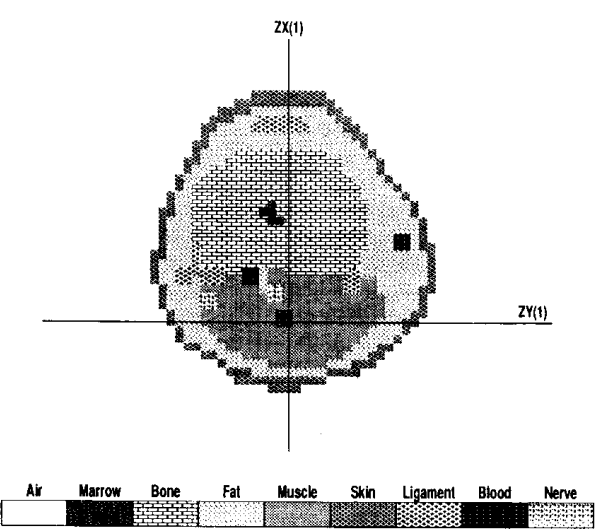

Tissue in $\mathrm{XY}(3)$ cross-section

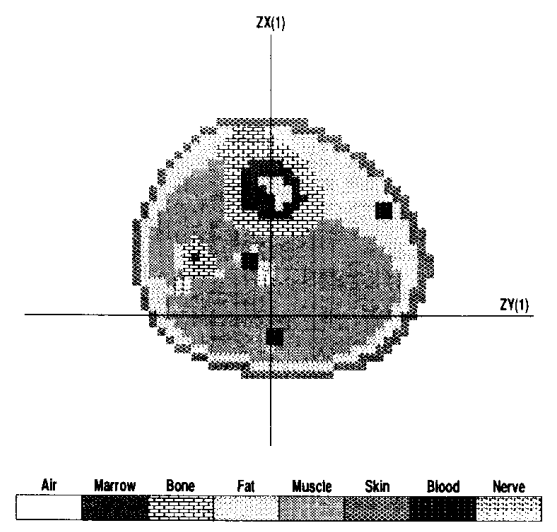

Tissue in $\mathrm{XY}(4)$ cross-section
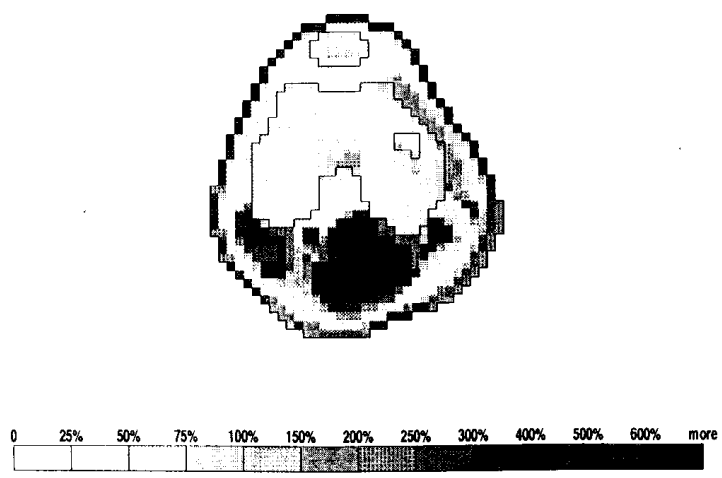

EM power distribution in $\mathrm{XY}(2)$ cross-section

(d)
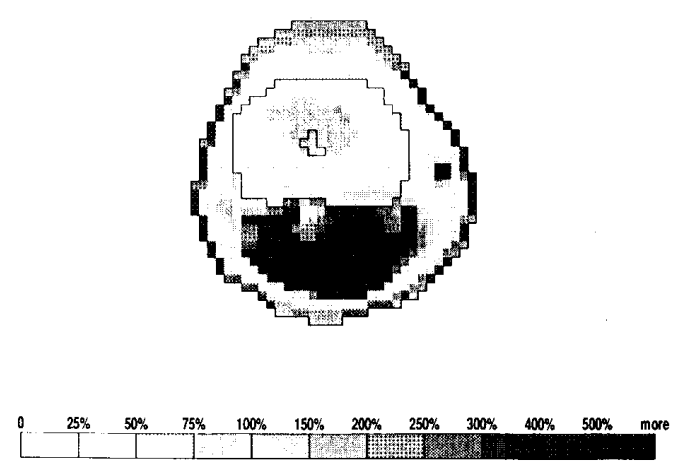

EM power distribution in $\mathrm{XY}(3)$ cross-section (e)

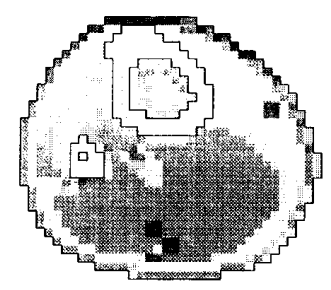
(f)

EM power distribution in $\mathrm{XY}(4)$ cross-section

Fig. 4 (Continued) 
We are now able to determine the EM power distribution in realistic three-dimensional models, provided that the frequency of operation is such that the quasi-static approximation may be applied.

\section{ACKNOWLEDGMENT}

The authors are indebted to G. van Rhoon from Dr. D. Den Hoed Kliniek in Rotterdam, The Netherlands, for a help with the knee-model.

\section{REFERENCES}

[1] G. C. van Rhoon, A. G. Visser, P. M. van den Berg, and H. S. Reinhold, "Evaluation of ring capacitor plates for regional deep heating," Int. J. Hyperthermia, vol. 4, pp. 133-142, 1988.

[2] M. J. Sowinski, M. H. P. M. van Putten, P. M. van den Berg, and G. C. van Rhoon, "A quasi-static model for the ring capacitor applicator," IEEE Trans. Biomed. Eng., vol. 36, pp. 995-1003, 1989.

[3] N. N. Yanenko, The Method of Fractional Steps. Berlin: Springer, 1971

[4] A. R. Mitchell and D. F. Griffiths, The Finite Difference Method in Partial Differential Equations. New York: Wiley, 1980

[5] R. S. Ledley, H. K. Huang, and J. C. Mazziotta, Cross-Sectional Anatomy-An Atlas for Computerized Tomography. Baltimore, MD: Waverly, 1977.

[6] H. O. Kendall, F. P. Kendall, and G. E. Wadsworth, Muscles, Testing and Function. Baltimore, MD: Williams and Wilkins, 1971

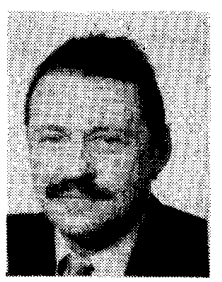

Maciej J. Sowiński was born in Warsaw, Poland, on July 24,1947 . He received the M.S. degree with first class honors in mathematics from Warsaw University of Technology in 1971 and the $\mathrm{Ph} . \mathrm{D}$. degree in 1980 from the Institute of Fundamental Technological Research, Warsaw, Poland.

In 1972 he joined the Department of Electromagnetic Waves Theory, Institute of Fundamental Technological Research, Warsaw, Poland where he was appointed as an Assistant Professor. He has been engaged in research on waveguides and microwave devices, dielectric optical waveguides, and applications of null-field method and eigenfunction expansion method to field problems. From 1987 to 1989 he was a research fellow in the Laboratory of Electromagnetic Research, Delft University of Technology, Delft, The Netherlands, where he was working on computational methods for hyperthermic applications. At present, he is working in the field of electromagnetic inductive excitation with application to the physiotherapy.

Dr. Sowinski is a member of the Association of Polish Electronic Engineers.

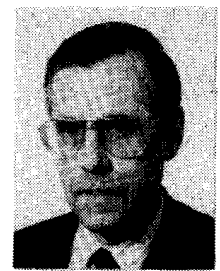

Peter M. van den Berg was born in Rotterdam, The Netherlands, on November 11, 1943. He received the degree in electrical engineering from the Polytechnical School of Rotterdam in 1964, the B.Sc. and M.Sc. degrees in electrical engineering, and the Ph.D. degree in technical sciences, all from the Delft University of Technology, Delft, The Netherlands, in 1966, 1968, and 1971 , respectively.

From 1967 to 1968 , he was employed as a $\mathrm{Re}$ search Engineer by the Dutch Patent Office. Since 1968, he has been a member of the Scientific Staff of the Electromagnetic Research Group of the Delft University of Technology. During these years, he carried out research and taught classes in the area of wave propagation and scattering problems. During the academic year 1973-1974 he was a Visiting Lecturer in the Department of Mathematics, University of Dundee, Scotland. During a three-month period in 1980-1981, he was a Vis iting Scientist at the Institute of Theoretical Physics, Goteborg, Sweden He was appointed Professor at the Delft University of Technology in 1981. At present, his main research interest is the efficient computation of field problems using iterative techniques based on error minimization and the computation of fields in strongly inhomogeneous media. At the moment he is acting Head of the Laboratory of Electromagnetic Research of the Delft University of Technology. 